\title{
MHC-I Ligand Discovery Using Targeted Database Searches of Mass Spectrometry Data: Implications for T-Cell Immunotherapies
}

Murphy, J. Patrick; Konda, Prathyusha; Kowalewski, Daniel J.; Schuster, Heiko; Clements, Derek; Kim, Youra; Cohen, Alejandro M.; Sharif, Tanveer; Nielsen, Morten; Stevanovic, Stefan

Total number of authors:

12

Published in:

Journal of Proteome Research

Link to article, DOI:

10.1021/acs.jproteome.6b00971

Publication date:

2017

Document Version

Publisher's PDF, also known as Version of record

Link back to DTU Orbit

Citation (APA):

Murphy, J. P., Konda, P., Kowalewski, D. J., Schuster, H., Clements, D., Kim, Y., Cohen, A. M., Sharif, T., Nielsen, M., Stevanovic, S., Lee, P. W., \& Gujar, S. (2017). MHC-I Ligand Discovery Using Targeted Database Searches of Mass Spectrometry Data: Implications for T-Cell Immunotherapies. Journal of Proteome Research, 16(4), 1806-1816. https://doi.org/10.1021/acs.jproteome.6b00971

\section{General rights}

Copyright and moral rights for the publications made accessible in the public portal are retained by the authors and/or other copyright owners and it is a condition of accessing publications that users recognise and abide by the legal requirements associated with these rights.

- Users may download and print one copy of any publication from the public portal for the purpose of private study or research.

- You may not further distribute the material or use it for any profit-making activity or commercial gain

- You may freely distribute the URL identifying the publication in the public portal 


\section{proteome \\ MHC-I Ligand Discovery Using Targeted Database Searches of Mass Spectrometry Data: Implications for T-Cell Immunotherapies}

J. Patrick Murphy, ${ }^{\dagger}, \bigcirc$ Prathyusha Konda, ${ }^{\dagger, \bigcirc}$ Daniel J. Kowalewski, ${ }^{\ddagger \S}$ Heiko Schuster, ${ }^{\ddagger \S}$ Derek Clements, Youra Kim,, Alejandro M. Cohen, ${ }^{\perp}$ Tanveer Sharif, ${ }^{\dagger}$ Morten Nielsen, ${ }^{\#}$ Stefan Stevanovic, ${ }^{\ddagger}$ Patrick W. Lee, ${ }^{\dagger}, \|$ and Shashi Gujar ${ }^{*},+,, \nabla \odot$

${ }^{\dagger}$ Department of Microbiology and Immunology, Dalhousie University, Halifax, Nova Scotia B3H 4R2, Canada

${ }^{\ddagger}$ Department of Immunology, Interfaculty Institute for Cell Biology, University of Tübingen, 72076 Tübingen, Germany

${ }^{\S}$ Immatics Biotechnologies GmbH, 72076 Tübingen, Germany

"Department of Pathology, Dalhousie University, Halifax, Nova Scotia B3H 4R2, Canada

${ }^{\perp}$ Proteomics Core Facility, Dalhousie University, Halifax, Nova Scotia B3H 4R2, Canada

\# Department of Bio and Health Informatics, Technical University of Denmark, 2800 Kongens Lyngby, Denmark

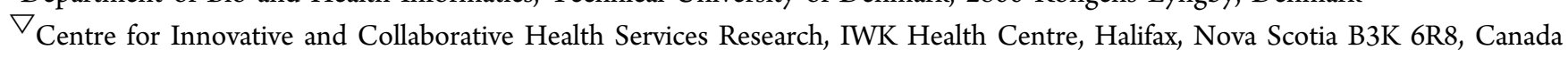

Supporting Information

ABSTRACT: Class I major histocompatibility complex (MHCI)-bound peptide ligands dictate the activation and specificity of $\mathrm{CD}^{+} \mathrm{T}$ cells and thus are important for devising $\mathrm{T}$-cell immunotherapies. In recent times, advances in mass spectrometry (MS) have enabled the precise identification of these MHC-I peptides, wherein MS spectra are compared against a reference proteome. Unfortunately, matching these spectra to reference proteome databases is hindered by inflated search spaces attributed to a lack of enzyme restriction in the searches, limiting the efficiency with which MHC ligands are discovered. Here we offer a solution to this problem whereby we developed

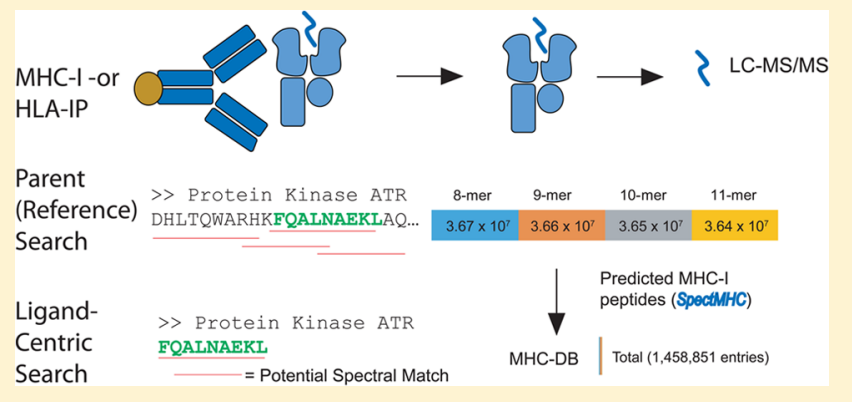
a targeted database search approach and accompanying tool SpectMHC, that is based on a priori-predicted MHC-I peptides. We first validated the approach using MS data from two different allotype-specific immunoprecipitates for the C57BL/6 mouse background. We then developed allotype-specific HLA databases to search previously published MS data sets of human peripheral blood mononuclear cells (PBMCs). This targeted search strategy improved peptide identifications for both mouse and human ligandomes by greater than 2 -fold and is superior to traditional "no enzyme" searches of reference proteomes. Our targeted database search promises to uncover otherwise missed novel T-cell epitopes of therapeutic potential.

KEYWORDS: mass spectrometry, MHC ligandome, CD8 T-cell epitopes, immunotherapy, database searching

\section{INTRODUCTION}

Antigen-specific activation of $\mathrm{CD}^{+} \mathrm{T}$-cells is initiated when a specific T-cell receptor recognizes its cognate peptide epitopes presented through class I major histocompatibility complexes (MHC-I) on antigen presenting cells. These MHC-bound peptides, collectively known as the MHC-I ligandome, are usually 8-11 amino acids long and are derived from intracellular-cleaved "self" or foreign proteins. ${ }^{1}$ MHC-I molecules, termed Human Leukocyte Antigens (HLA) in humans, are highly polymorphic, particularly in the human population, and the peptides bound are highly dependent on an individual's HLA allotype. Surveying the MHC-I ligandome in both a context- and allotype-specific manner is of the utmost importance in understanding how the adaptive immune response is regulated in pathogen infection and cancer. Furthermore, precise MHC-I peptide identification from mass spectrometry (MS) data is especially important in designing therapeutic targets that could be used to formulate personalized $\mathrm{CD} 8^{+} \mathrm{T}$-cell-based therapies such as vaccines against infectious diseases. ${ }^{2}$ Additionally, for vaccine-based cancer immunotherapies, MS-enabled identification of tumor-associated MHC-I ligands or those containing neo-antigens is crucial.

Identifying MHC-I ligands by MS remains difficult due to low peptide abundance, but advancements in MS sensitivity are revolutionizing the process. Currently, the main experimental approach is to perform immuno-precipitation (IP) of MHC-I proteins, followed by peptide elution, purification, and analysis by LC-MS/MS. ${ }^{5}$ For mouse models, well-characterized MHCI allotype-specific antibodies can be employed in the IP because allelic diversity in mice is low. In contrast, human allelic diversity is high but pan-HLA-specific antibodies such as the

Received: November 15, 2016

Published: February 28, 2017 
W6/32 antibody are well established. ${ }^{6}$ Spectra from the antibody eluates are then scored against a protein database using search algorithms such as Mascot and filtered to a certain false discovery rate (FDR). Following the database searches, it is common for a large portion of the collected spectra to not be assigned a peptide identification. Recently, this has been partially attributed to unforeseen proteasomally spliced peptides nonexistent in the database searches. ${ }^{1}$ In the past, other types of nonstandard MHC-I peptides have also been reported that may explain missing spectral assignments in database searches such as noncanonical reading frames ${ }^{7}$ and nonclassical peptide cleavage. ${ }^{8}$ However, another common problem that complicates spectral assignments in all MHC ligandome analyses to date is the inability to narrow the number of potential spectral matches by specifying a proteolytic enzyme cleavage specificity, as would be implemented when searching shotgun proteomics data. As a result, vast search spaces are explored during database searches of MHC-I IP MS data because the composition of peptide assignments tested is not representative of the sample. As such, statistical power is low when estimating FDRs for MS/MS spectra. It has been proposed for proteomics studies that search space sizes could be reduced by limiting them to only those peptides likely to be present in the sample. ${ }^{9,10}$ This strategy would improve FDR estimations and increase the number of peptides identified from shotgun MS data. We therefore questioned whether the search spaces for MHC-I IP MS database searches could be limited solely to predicted MHC-I ligands for the allotypes of the sample to improve identification rates.

Various MHC-I peptide prediction tools are available to implement the MHC-peptide database search approach. ${ }^{11-14}$ Most predictors are trained on existing MHC-I binding assay data and output lists of potential MHC-I peptides from protein sequences. ${ }^{15}$ One such tool, NetMHC, ${ }^{12}$ uses neural networks trained on large data sets of MHC-I peptide binding affinity values and predicts probable MHC-I ligands for queried proteins. Executing NetMHC in mouse models is practical because few allotypes exist among mouse MHC-I proteins. Although polymorphism of human alleles is high, performing HLA predictions is now becoming feasible as peptide-binding data sets are being collected on more allotypes. ${ }^{16}$

Here we developed a targeted database search strategy using NetMHC predictions to compile peptide databases to search MHC-I IP MS data. The approach leads to greatly improved numbers of MHC class I peptide identifications from both murine and human IPs. To implement the targeted searches, we made an accompanying Python-based tool, SpectMHC, available to the immunology community. On the basis of a reference proteome, SpectMHC compiles targeted MHC-I peptide FASTA databases to be used for searching MHC-I IP MS data and is available on GitHub: https://github.com/ Prathyusha-konda/SpectMHC. It is worth noting that our approach not only is applicable to current and future MHC ligand discovery efforts but also can be used retrospectively; when we applied it to already published MHC ligandome data sets, MHC ligands were detected that otherwise went unreported.

\section{EXPERIMENTAL SECTION}

\section{Cell Culture}

EL4 cells were originally purchased from ATCC and were maintained in Dulbecco's modified Eagle medium (Invitrogen,
Waltham, MA), containing 10\% FBS (Invitrogen) and 5\% antibiotic/antimycotic (Invitrogen). Cells were grown in T75 flasks to a density of $2 \times 10^{6}$ cells $/ \mathrm{mL}$ before subculturing or harvesting for experiments.

\section{Mouse MHC-I and Human HLA Peptide Database Compilation}

Mouse and human FASTA databases were downloaded from UniProtKB (mouse, December 2015; human, February 2015), containing all mouse or human proteins including isoforms and variants. Using an offline version of NetMHC (version 4.0), allotype-specific mouse or human binding affinities were predicted for all 8-, 9-, 10-, and 11-mer peptides for 2 mouse allotypes $\left(\mathrm{H}-2 \mathrm{D}^{\mathrm{b}}\right.$ and $\left.\mathrm{H}-2 \mathrm{~K}^{\mathrm{b}}\right)$ and 14 human allotypes $\left(\mathrm{A}^{*} 01\right.$, $A^{*} 02, A^{*} 03, A^{*} 11, A^{*} 24, A^{*} 29, A^{*} 32, B^{*} 07, B^{*} 08, B^{*} 27$, $B * 35, B^{*} 39, B^{*} 44$, and $\left.B * 51\right)$. All peptides with NetMHCpredicted binding affinities $\leq 2 \%$ rank for each allotype were then compiled into a new FASTA database. All database manipulations were performed using Python and R scripts.

\section{MHC-I Peptide Immuno-Precipitation}

An aliquot of $8 \times 10^{8}$ EL4 cells was pelleted and used for MHC-I peptide immuno-precipitation as previously described. ${ }^{17}$ In brief, cells were lysed in PBS containing $0.4 \%$ CHAPS and mini-complete protease inhibitor tablets (Roche, Indianapolis, IN). Antibodies were produced in-house from hybridoma clones B22.249 (H-2 $\mathrm{D}^{\mathrm{b}}$-specific) and $\mathrm{Y} 3\left(\mathrm{H}-2 \mathrm{~K}^{\mathrm{b}}\right.$ specific). MHC-I proteins were precipitated from the cell lysate using $2 \mathrm{mg}$ of each antibody coupled to $80 \mathrm{mg}$ of $\mathrm{CNBr}$ activated Sepharose 4B resin (Uppsala, Sweden). Incubations were performed overnight at $4{ }^{\circ} \mathrm{C}$ in $10 \mathrm{~mL}$ glass tubes. Bound MHC-I proteins and peptides were washed with $40 \mathrm{~mL}$ of PBS, then $30 \mathrm{~mL}$ of Milli-Q water, and then eluted eight times with $200 \mu \mathrm{L}$ of $0.2 \%$ trifluoroacetic acid (TFA). Eluates were filtered through $3 \mathrm{kDa}$ molecular weight cutoff filters (Millipore, Cork, Ireland); then, the filtrate was lyophilized and desalted using Stage-tips. ${ }^{18}$

\section{Mass Spectrometry}

Lyophilized peptides were solubilized in $12 \mu \mathrm{L}$ of $1 \%$ formic acid and analyzed by LC-MS/MS. For each antibody eluate, an aliquot of $1 \mu \mathrm{L}$ of peptides was injected onto a $75 \mu \mathrm{m} \times 30 \mathrm{~cm}$ column (New Objective, Woburn, MA) self-packed with $4 \mu \mathrm{m}$, $90 \AA ̊$, Proteo C18 material (Phenomenex, Torrance, CA). Online chromatography was performed using a Dionex Ultimate 3000 UHPLC (Thermo-Scientific, San Jose, CA) at a flow rate of $300 \mathrm{~nL} / \mathrm{min}$. Peptides were separated and eluted into the mass spectrometer using a gradient of 3 to $35 \%$ acetonitrile ( $0.1 \%$ formic acid) over $65 \mathrm{~min}$, followed by $5 \mathrm{~min}$ at $95 \%$ acetonitrile ( $0.1 \%$ formic acid). MS was performed using an Orbitrap Velos Pro (Thermo-Scientific, San Jose, CA) operated in data-dependent mode. Survey scans (MS1) were performed using the Orbitrap over a scan range of 350-650 m/ $z$ and resolution setting of 60000 . A lock mass of 445.12003 $\mathrm{m} / z$ was used to achieve internal mass calibration as previously described. ${ }^{19}$ On the basis of MS1 scans, MS2 scans were performed using the ion trap, selecting the top 10 most intense precursor (MS1) ions for fragmentation by collision-induced dissociation (CID) at $35 \%$ collision energy with a precursor isolation window of $2 \mathrm{~m} / z$. MS2 scans were only collected on peptides with charge states of $2^{+}$or $3^{+}$with a minimum MS1 intensity of 50 counts. Advanced gain control (AGC) settings were $5 \times 10^{5}$ for Orbitrap scans and $2 \times 10^{5}$ for ion trap scans. 
A

Parent-DB: No enzyme search

$>$ Protein Kinase ATR DHLTQWARHKFQALNAEKLAQ...

MHC-DB: Peptide-centric, No cleavage search

>> Protein Kinase ATR

FQALNAEKL

$=$ Potential Spectral Match

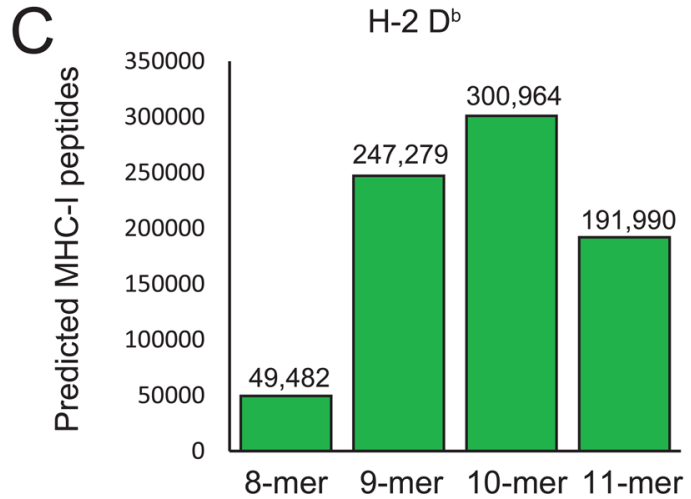

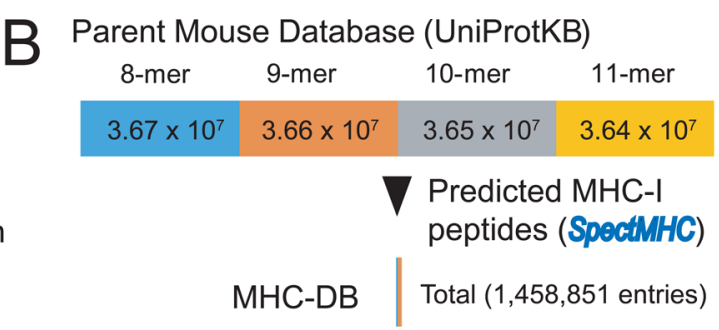

$\mathrm{H}-2 \mathrm{~K}^{\mathrm{b}}$

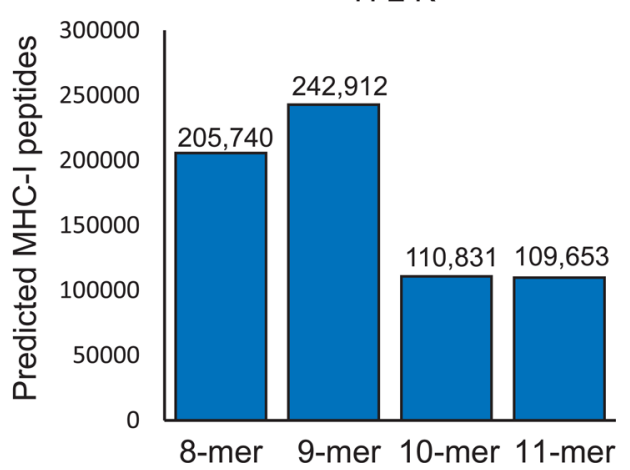

Figure 1. Predicting MHC-I peptides for the full mouse proteome. (A) Targeted MHC-I search (MHC-DB), performed with no cleavage specificity, limits potential spectral matches and decreases the search space compared with a reference proteome (Parent-DB) search. (B) Beginning with a reference database of mouse proteins, a targeted MHC-I- database (MHC-DB) solely containing predicted sequences was formed (NetMHC $\leq 2 \%$ rank) using SpectMHC. The MHC-DB contained peptides from the H-2 $\mathrm{D}^{\mathrm{b}}$ and $\mathrm{H}-2 \mathrm{~K}^{\mathrm{b}}$ mouse alleles. Shown are the numbers of 8- to $11-\mathrm{mer}$ peptide sequences in the parent database and the total NetMHC-predicted peptides $\left(\mathrm{H}-2 \mathrm{D}^{\mathrm{b}}\right.$ and $\left.\mathrm{H}-2 \mathrm{~K}^{\mathrm{b}}\right)$. (C) Distribution of the total NetMHCpredicted ( $\leq 2 \%$ rank) mouse MHC-I peptides (by 8- to 11 -mers) for the $\mathrm{H}-2 \mathrm{D}^{\mathrm{b}}$ and $\mathrm{H}-2 \mathrm{~K}^{\mathrm{b}}$ allotypes used to create the MHC-DB.

\section{Database Searching and Comparisons}

Mouse and human database searches were performed using either Sequest or Mascot implemented with Proteome Discoverer 1.4. The parent ion (MS1) tolerance was $5 \mathrm{ppm}$ and fragment ion tolerance was $0.5 \mathrm{Da}$. Spectra for each IP were searched against either the parent database (Parent-DB) or the MHC-targeted database (MHC-DB or HLA-DB). Spectra were searched against Parent-DB using no enzyme specificity but searched against MHC-DB or HLA-DB using no cleavage restriction. Peptide FDR was controlled using Percolator. All peptides with "medium" (5\% FDR) or "high" (1\% FDR) confidence were reported in the final data sets. Peptide and Peptide Spectral Match (PSM) analyses comparing results for the different antibody eluates and database searches were performed using R. Mouse MS (.raw) files have been deposited to Chorus, https://chorusproject.org, ID\#1098.

Code Availability

We have made SpectMHC available on GitHub: https://github. com/Prathyusha-konda/SpectMHC. This semicommand-linebased tool is customized to be used with preinstalled standalone NetMHC packages, in Darwin (Mac) and Linux platforms. The tool takes an input protein FASTA database and outputs predicted MHC-binding peptides using NetMHC 4.0, NetMHC 3.4, or NetMHCpan. The instructions to utilize the code are available in the readme file found in the above GitHub folder.

\section{RESULTS AND DISCUSSION}

\section{Generation of Targeted MHC-I Databases}

Improving MHC peptide ligand identification and measurement by MS is important because MHC-I peptides are not produced from easily predictable enzymatic cleavage sites. This leads to all peptides within a specific precursor ion tolerance being tested, resulting in large search spaces that most likely result in inflated FDR statistics and low specificity. It has been proposed that the assignment of spectra from shotgun MS experiments can be improved by tailoring the search database to those peptides likely to be in the sample. ${ }^{9,20}$ Even in the proteomics field, recent attempts have been made to narrow search spaces to improve protein identifications from peptide digests by, for example, using databases formed from spectral libraries of previously identified peptides ${ }^{10}$ or from RNASeq data collected from the samples. ${ }^{21}$ In these cases, the targeted searches resulted in databases that more accurately reflected the samples analyzed and increased the number of identified peptides. We thus anticipated that this targeted strategy could be employed to reduce the complexity of databases used for MHC-I IP MS searches. Searches against a targeted MHC-I peptide database could then be implemented in which the MHC prediction tool replaces enzymatic cleavage specificity. Such a strategy allows the use of a no cleavage search, whereby spectra are only tested against full database entries and not to non-MHC peptides residing in the sequences therein. This is exemplified by the predicted MHC peptide, FQALNAEKL from the mouse protein kinase ATR (Figure 1A), where instead of the many matches to the full protein when using a no enzyme search, a no cleavage search of the targeted database 

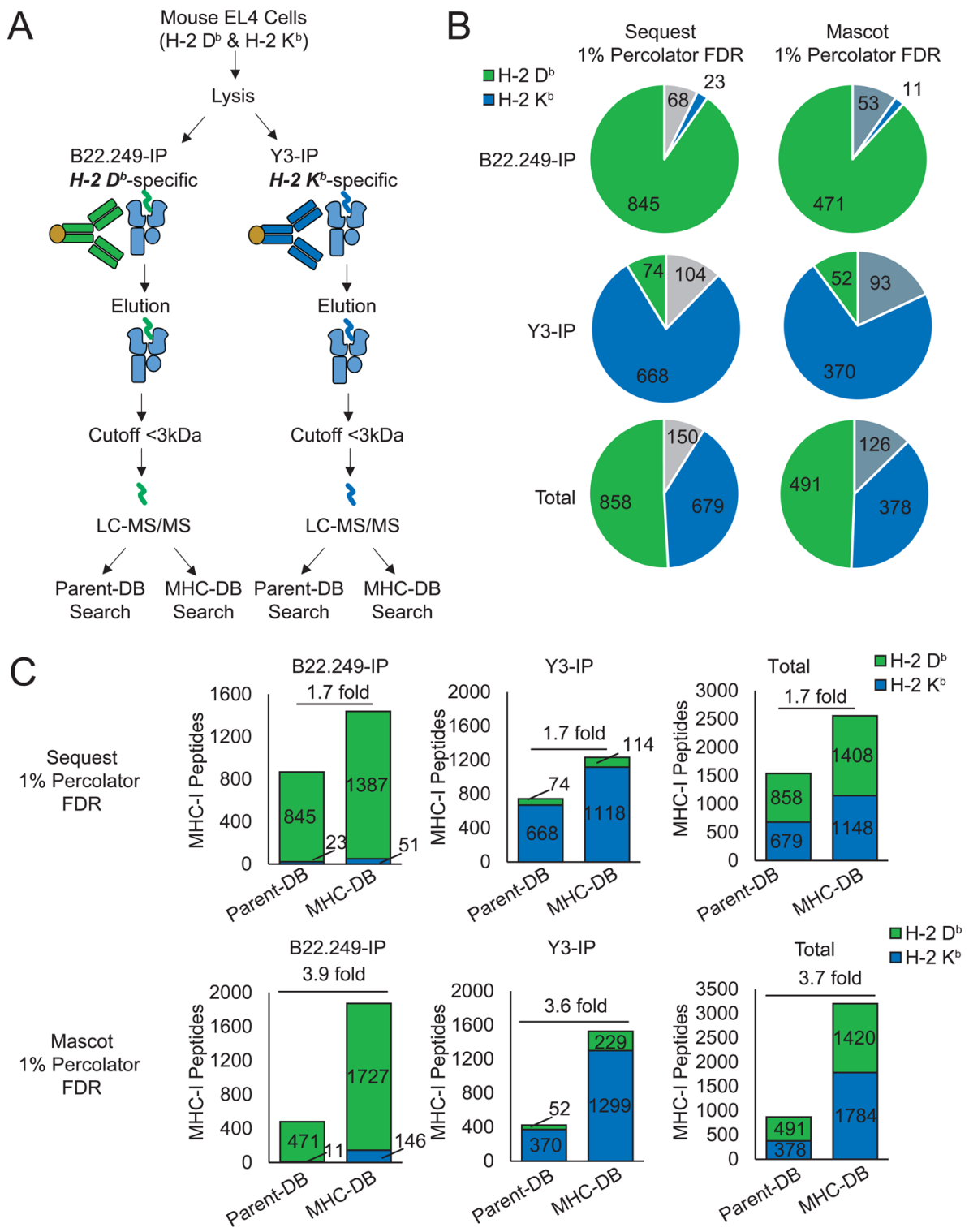

Figure 2. Targeted MHC-I peptide database search increases MHC ligandome identifications. (A) Schematic for an allotype-specific, two-antibody experiment to assess the MHC-DB search. $\mathrm{H}-2 \mathrm{D}^{\mathrm{b}}$ and $\mathrm{H}-2 \mathrm{~K}^{\mathrm{b}}$-specific antibodies (B22.249 and $\mathrm{Y} 3$, respectively) were used to immuno-precipitate MHC-I proteins from mouse EL4 cells (carrying the $\mathrm{H}-2 \mathrm{D}^{\mathrm{b}}$ and $\mathrm{H}-2 \mathrm{~K}^{\mathrm{b}}$ allotypes), followed by elution of peptides, separation from MHC-I proteins by a cutoff filter, and analysis by LC-MS/MS. Spectra were assigned using Sequest and Mascot and false discovery rates (FDRs) determined using Percolator with either the Parent-DB or MHC-DB. (B) Parent-DB search results for mouse EL4 cells at 1\% FDR, showing purity and allotype specificity of the B22.249 and Y3 antibodies. Shown separately for each allele-specific IP (and the total of both) are the number of unique peptides considered $\mathrm{H}-2 \mathrm{D}^{\mathrm{b}}$ binders (NetMHC $\leq 2 \%$ rank for $\mathrm{H}-2 \mathrm{D}^{\mathrm{b}}$ ), $\mathrm{H}-2 \mathrm{~K}^{\mathrm{b}}$ binders (NetMHC $\leq 2 \%$ rank for H-2 $\mathrm{K}^{\mathrm{b}}$ ), or nonbinders $(\mathrm{NetMHC}>2 \%$ rank, gray). (C) Comparison of the MHC-I peptides (NetMHC $\leq 2 \%$ rank) identified using the targeted MHC-DB search approach with both Sequest and Mascot compared to the no enzyme Parent-DB search (1\% FDR).

results in fewer potential matches to unlikely spectral assignments.

We began with a simple mouse model for which the MHC-I peptides could be easily predicted due to their low MHC-I allelic polymorphism. The commonly used C57BL/6 laboratory mouse strain carries the well-characterized $\mathrm{H}-2 \mathrm{D}^{\mathrm{b}}$ and $\mathrm{H}-2 \mathrm{~K}^{\mathrm{b}}$ mouse MHC-I allotypes, for which hybridomas producing allele-specific antibodies exist. To generate a targeted MHC-I database, we used a "parent" reference database containing 56479 mouse UniProtKB protein sequences (Parent-DB). Although many other tools are available such as SYFPEITHI $^{14}$ and IEDB, ${ }^{13}$ we have employed NetMHC for peptide predictions because it has high allotype coverage.
Furthermore, in situations where the HLA alleles in question are not covered by NetMHC 4.0, the pan-allotype-specific NetMHCpan 3.0 predictor performs very well. ${ }^{22}$ Using NetMHC (offline, version 4.0), we predicted the $\mathrm{H}-2 \mathrm{D}^{\mathrm{b}}$ and $\mathrm{H}-2 \mathrm{~K}^{\mathrm{b}}$ binding affinities for all possible 8-, 9-, 10-, and 11-mer peptides for each protein in the parent database (Figure 1B). Rather than a specific nM affinity cutoff, a \% rank cutoff in NetMHC has recently been shown to be more versatile across different allotypes, whereby the top $2 \%$ ranking predicted $\mathrm{MHC}$ peptides show high specificity. ${ }^{22}$ Therefore, all 8 - to 11-mer peptides were filtered to $\leq 2 \%$ NetMHC rank. This vastly smaller list of peptides was compiled into a targeted, MHC-I peptide database (MHC-DB) (Figure 1B). The MHC-DB is 
composed of 1458851 entries of which $\mathrm{H}-2 \mathrm{D}^{\mathrm{b}}$ peptides were $38 \% 8$ - to 9-mers whereas $\mathrm{H}-2 \mathrm{~K}^{\mathrm{b}}$ peptides were $67 \% 8$ - to 9mers (as illustrated in Figure 1C). The MHC-DB contained only $1 \%$ of the 8 - to 11 -mers in the Parent-DB (Figure 1B); thus the potential peptide identifications in the search space decreased by $\sim 2$ orders of magnitude. It is clear that MHC-I searches against this database would successfully limit the search space to a small fraction of the mouse proteome. Using the database for searching MS data also replaces the need for an enzymatic cleavage specificity because it is inherently built into the database prediction step. The targeted database allows for a novel, simplified search strategy for MHC-I database searches to increase ligand identification. Because it would be of interest to others, we offer a supporting tool (SpectMHC) to make searchable targeted MHC-I FASTA databases.

\section{Targeted MHC-I Peptide Database Searches Increase} Identification Rates for MHC-I Ligands

With the mouse MHC-I peptide FASTA database in hand, we then empirically tested the targeted MHC-I database search strategy. As a model, we used the EL4 mouse cell line (C57BL/ 6 background) and performed allotype-specific MHC-IP experiments using established protocols. ${ }^{17}$ Specificity was enabled using antibodies specific to the H-2 $\mathrm{D}^{\mathrm{b}}$ (B22.249 hybridoma ${ }^{23}$ ) or $\mathrm{H}-2 \mathrm{~K}^{\mathrm{b}}$ (Y3 hybridoma ${ }^{24}$ ) mouse allotypes (Figure 2A). Following antibody-based MHC molecule elution and peptide separation, peptide mass spectra were generated using shotgun LC-MS/MS. An issue with limiting the search space only to MHC-I peptides is that non-MHC peptides may be falsely matched to MHC-I peptides because non-MHC matches are not present. To test this, we intentionally did not separate NetMHC-predicted $\mathrm{H}-2 \mathrm{D}^{\mathrm{b}}$ and $\mathrm{H}-2 \mathrm{~K}^{\mathrm{b}}$ predictions. This strategy allowed us to assess whether non-MHC peptides would be matched to the MHC-DB because correct peptide matches from each IP should reflect the specificity of the antibody used. Alternatively, if peptide matches to the MHCDB search were incorrect, then new peptide assignments should be equally distributed among $\mathrm{H}-2 \mathrm{D}^{\mathrm{b}}$ and $\mathrm{H}-2 \mathrm{~K}^{\mathrm{b}}$ peptides independent of the antibody.

To estimate false discovery rates for peptide identification, commercial software implementations of tools such as Percolator ${ }^{25}$ are common and use reversed or shuffled versions of the peptides as decoy databases. Using the MHC-I peptidetargeted approach, a decoy database of reversed peptides can be formed either before or after NetMHC prediction. Reversing proteins before predicting requires an additional round of NetMHC prediction and requires specifying target and decoy databases during the search, which in most commercial database search tools is not yet implementable. Before implementing our searches with commercial software (Proteome Discoverer) we manually assessed the target decoy strategy, comparing Sequest search PSMs where the decoy was formed by (1) reversing proteins in the Parent-DB then predicting by NetMHC (Reverse then Predict) or (2) reversing the NetMHC-predicted peptides (Predict then Reverse). We observed the overall distribution (by Sequest XCorr) of target and decoy hits (PSMs) to be similar by both strategies for both the B22.249 and Y3 IPs (Supplementary Figure 1A). Furthermore, in our 2-antibody model both approaches accurately returned target PSMs of the correct antibody ( $\mathrm{H}-2$ $\mathrm{D}^{\mathrm{b}}$ to $\mathrm{B} 22.249$ and $\mathrm{H}-2 \mathrm{~K}^{\mathrm{b}}$ to $\mathrm{Y} 3$ ), whereas decoy PSMs were equally represented by the correct or incorrect allele-matching PSMs (Supplementary Figure 1B). Manual estimation of the
FDR as previously described ${ }^{26}$ showed only minor differences in the total number of unique peptides from each target decoy strategy, and antibody allotype was conserved at 5\% (Supplementary Figure 1C) and 1\% FDR (Supplementary Figure 1D). As such, the approach could be implemented using a single forward NetMHC-predicted database, which allowed implementing Percolator in Proteome Discoverer whereby reversed MHC peptides served as the decoy database.

To assess the EL4 MHC-I IP for the study, we first performed database searches against Parent DB using both Sequest and Mascot with no enzyme as the search specificity, with both 5\% and 1\% Percolator ${ }^{25}$ FDR thresholds. Using Sequest/Percolator at $1 \%$ FDR a total of 1537 unique peptides were identified between the B22.249 and Y3 antibody IPs. Using Mascot/Percolator at 1\% FDR, a total of 995 unique peptides were identified between the B22.249 and Y3 antibody IPs. Of these, only $8.9 \%$ (150) and $12.6 \%$ (126) of the Sequest and Mascot searches, respectively, were not considered MHC-I peptides using a 2\% NetMHC rank cutoff (Figure 2B). Furthermore, the IPs were allotype-specific, in that MHC-I peptides identified from the Sequest Parent-DB search were 97\% (845/868) H-2 $\mathrm{D}^{\mathrm{b}}$-specific and 90\% (668/742) H-2 $\mathrm{K}^{\mathrm{b}}$ specific (as revealed by a $2 \%$ NetMHC rank cutoff) for the B22.249 and Y3 IPs, respectively (1\% FDR) (Figure 2B). MHC-I peptides identified from the Mascot Parent-DB search were $98 \%(471 / 482) \mathrm{H}-2 \mathrm{D}^{\mathrm{b}}$-specific and $88 \%(370 / 422) \mathrm{H}-2$ $\mathrm{K}^{\mathrm{b}}$-specific for $\mathrm{B} 22.249$ and $\mathrm{Y} 3 \mathrm{IPs}$, respectively (1\% FDR) (Figure 2B). At 5\% Percolator FDR, a total of 2238 and 1942 peptides were identified by Sequest and Mascot searches, respectively, of which $250(11 \%)$ and $252(13 \%)$ were not considered MHC-I peptides by NetMHC ( $2 \%$ rank cutoff), respectively (Supplementary Figure $2 \mathrm{~A}$ ). Interestingly, even at this much less stringent FDR (5\%), antibody specificity was similarly maintained as it was at $1 \%$ FDR. MHC-I peptides identified from the Sequest Parent-DB search were 97\% (1062/ 1092) H-2 $\mathrm{D}^{\mathrm{b}}$-specific and $89 \%(894 / 1008) \mathrm{H}-2 \mathrm{~K}^{\mathrm{b}}$-specific (as revealed by a $2 \%$ NetMHC rank cutoff) for B22.249 and Y3 IPs, respectively (Supplementary Figure 2B). MHC-I peptides identified from the Mascot Parent-DB search were 98\% (911/ 934) $\mathrm{H}-2 \mathrm{D}^{\mathrm{b}}$-specific and $88 \%(747 / 850) \mathrm{H}-2 \mathrm{~K}^{\mathrm{b}}$-specific (as revealed by a $2 \%$ NetMHC rank cutoff) for B22.249 and Y3 IPs, respectively (Supplementary Figure 1B). Next, to test the targeted search approach, we performed Sequest and Mascot searches to match the MHC-IP spectra using no cleavage search specificity against the MHC-DB. These searches resulted in drastic improvements in unique MHC-I peptide identifications compared with the Parent-DB, specifically for the Mascot results: 3.9 and 3.6 fold for the B22.249 and Y3 IPs, respectively, and 3.7 fold across the total unique $\mathrm{H}-2 \mathrm{D}^{\mathrm{b}}$ and $\mathrm{H}$ $2 \mathrm{~K}^{\mathrm{b}}$ peptides identified ( $1 \%$ FDR in Figure 2C, 5\% FDR in Supplementary Figure 2B, Supplementary Data 1). Importantly, we find that Mascot searches with no cleavage also resulted in higher numbers of unique peptides compared with Sequest searches with no cleavage (Figure 2C, Supplementary Figure 2B). Matching non-MHC peptides to the MHC-DB was not evident based on maintenance of allotype-specificity, whereby 96 and $91 \%$ of the peptides identified from the Sequest MHCDB searches matched the appropriate antibody for the B22.249 $\left(\mathrm{H}-2 \mathrm{D}^{\mathrm{b}}\right.$-specific) and $\mathrm{Y} 3\left(\mathrm{H}-2 \mathrm{~K}^{\mathrm{b}}\right.$-specific) IPs, respectively (Figure 2C). For the Mascot searches, 90 and $84 \%$ of the peptides identified matched the appropriate antibody for the B22.249 and Y3 IPs, respectively (Figure 2C). As such, these peptides are assumed to be correct, and using the targeted 


$$
\begin{gathered}
A \\
\frac{0}{5} \\
\frac{1}{\pi} \\
\frac{1}{0} \\
\frac{0}{0} \\
\frac{0}{0} \\
\frac{0}{0} \\
\frac{0}{0} \\
0
\end{gathered}
$$
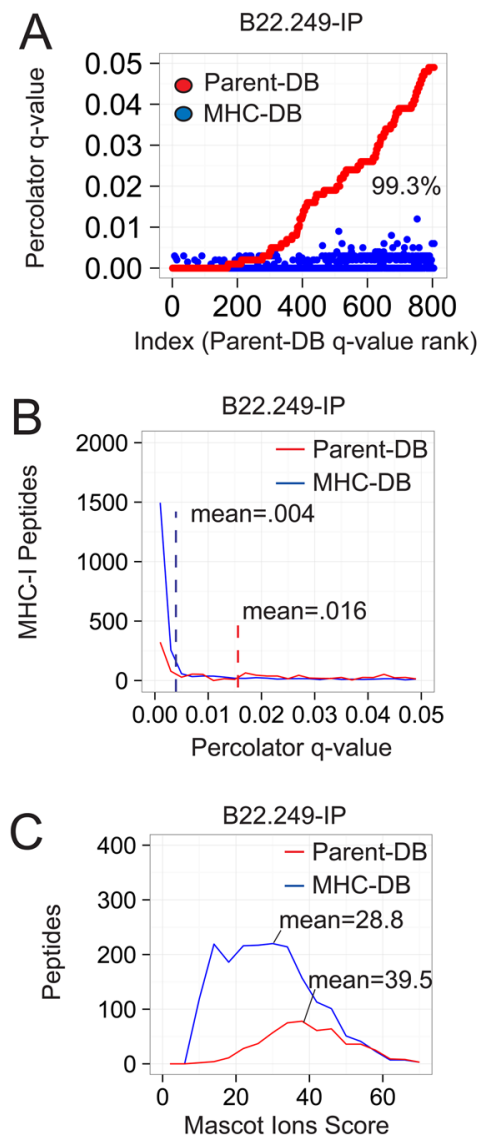
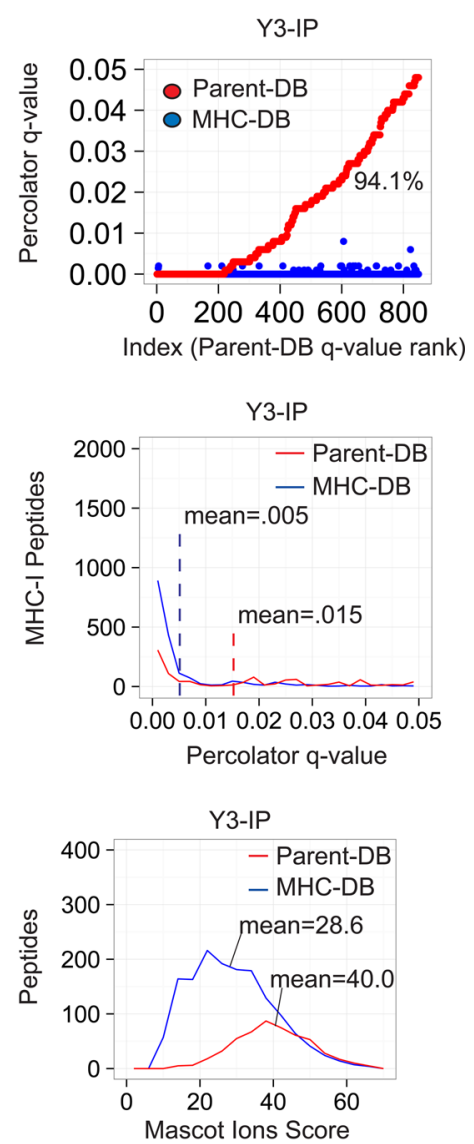

Figure 3. Effect of a targeted MHC database on search statistics. (A) Percolator $q$ values for each peptide identified in both search types are plotted in numerical order according to their Parent-DB $q$ values. Percentages are the peptides for which the $q$ value of the MHC-DB search was less than the Parent-DB search. (B) Distribution of the Percolator $q$ values (shown up to 5\% FDR) for each MHC-IP search result, comparing Parent-DB and MHC-DB search types. (C) Distribution and mean values of Mascot ions scores among identified peptides (1\% FDR) for the Parent-DB and MHCDB search types for the B22.249 and Y3 IPs.

search approach was successful. Accordingly, maintenance of allotype-specificity was also observed at 5\% FDR for both Sequest and Mascot (Supplementary Figure 2B). We also created MHC-DBs using 1, 5, and 10\% rank as NetMHC cutoffs, and a few additional peptides were identified with increased \% rank cutoffs (Supplementary Figure 3A,B). Further support of these MHC-I peptide assignments is evident from the distribution of binding affinities; 85 and $84 \%$ of Mascot MHC-DB search peptides were considered strong binders (NetMHC $\leq 0.5 \%$ rank) for the B22.249 H-2 $\mathrm{D}^{\mathrm{b}}$ and $\mathrm{Y} 3 \mathrm{H}-2$ $\mathrm{K}^{\mathrm{b}}$ peptides, respectively (Supplementary Figure $4 \mathrm{~A}$ ). These data are particularly supportive because the \% rank for MHCDB peptides $(\leq 2 \%)$ of all lengths was distributed relatively evenly (Supplementary Figure 4B). Taken together, these data show the potential for targeted database searches (particularly Mascot) in solving the "no enzyme" problem for MHC-I peptide identifications.

\section{Increased MHC-I Ligand Identifications Are Due to} Enhanced Statistical Power

In previous studies where the search space was reduced, increased numbers of identified peptides were mostly attributed to better statistical power when performing FDR estimation. ${ }^{20}$ Poor statistical power and sensitivity is especially a concern for searching MS data from MHC-I IPs using no enzyme restriction. In such searches, the likelihood of getting a highscoring incorrect match to a peptide not likely present in the sample is higher than with enzyme-restricted databases. As a result, false-positive estimates appear higher and fewer matches meet a required cutoff. In our mouse data, we examined our Mascot search results, which showed the greatest boost in performance, and we observed that when peptides identified in both the Parent-DB and MHC-DB search types are compared, 99.3 and $94.1 \%$ of Percolator $q$ values (FDR estimates) are lower for the MHC-DB search than the Parent-DB search for the B22.249 and Y3 IPs, respectively (Figure 3A). Percolator posterior error probabilities (PEPs), similar metrics to $q$ values, showed similar decreases (Supplementary Figure 5A). Furthermore, across all identified peptides, Percolator $q$ values and PEP were also distributed lower in the MHC-DB search (Figure 3B, Supplementary Figure 5B). Performing the MHCDB search thus improves statistical power for MHC IP searches, which, taken together with the aforementioned maintenance of allotype specificity, suggests that the approach is sensitive but also accurate. Furthermore, by performing the MHC-DB search, we were able to assign MS/MS spectra with lower Mascot ions scores (1\% FDR in Figure 3C, 5\% FDR in Supplementary Figure 6), which often occur when measuring peptides of low abundance such as MHC-I peptides. Interestingly, the approach also rescued the identification of many high Mascot-scoring spectra because they were identified only in the MHC-DB search (exemplified in Figure 4). As such, a mechanistic explanation for the improved peptide identification rates is the higher sensitivity occurring from decreasing the search space size to more accurately represent the sample. 
A

\section{B22.249-IP Peptides}

KQIANIDRI; lons Score 48.25, $\mathrm{H}-2$ D $\%$ rank 0.01
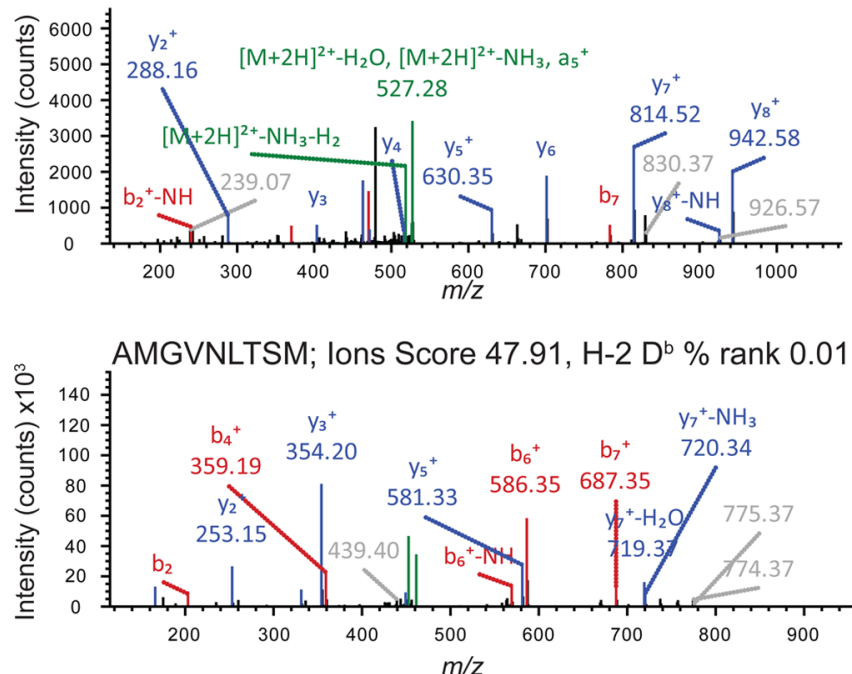

TGVINAPETI; Ions Score 47.56, H-2 D $\%$ rank 0.08

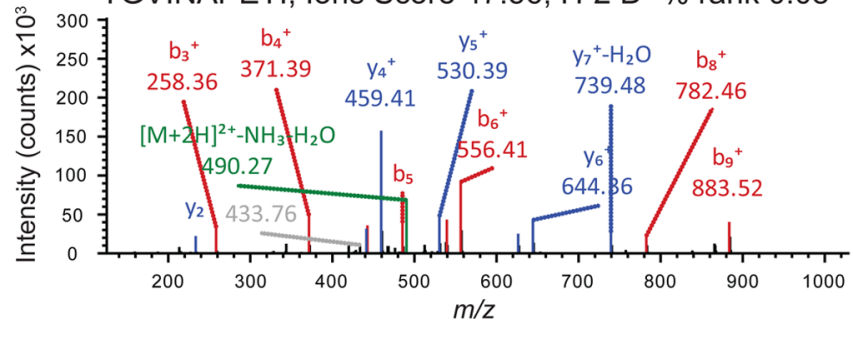

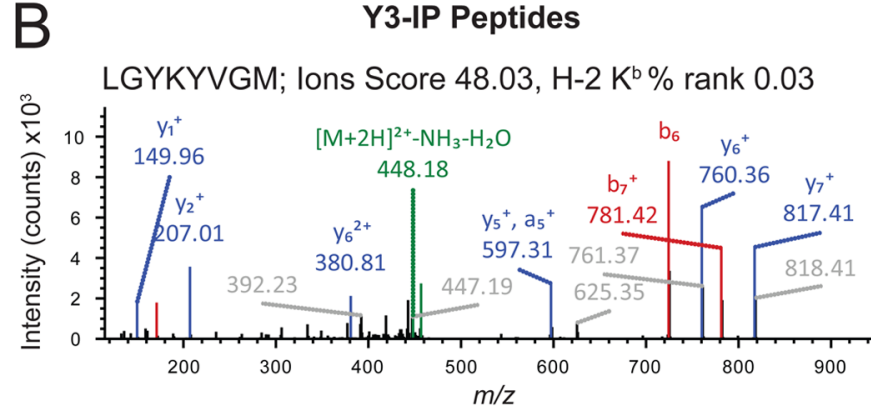

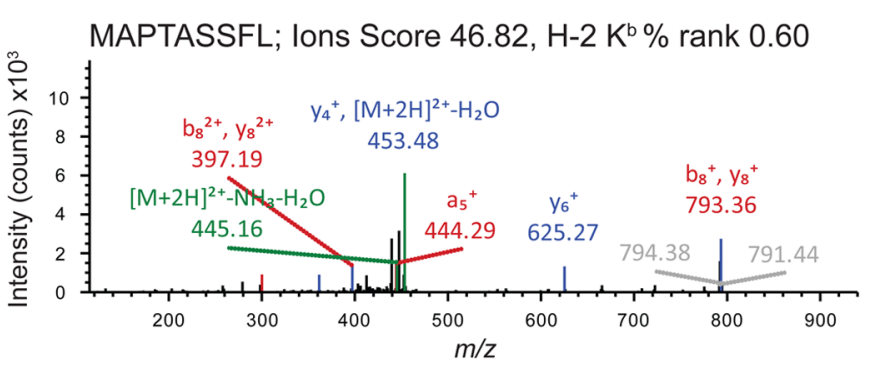

KVLVFSQM; Ions Score 46.82, H-2 K $\%$ rank 0.03

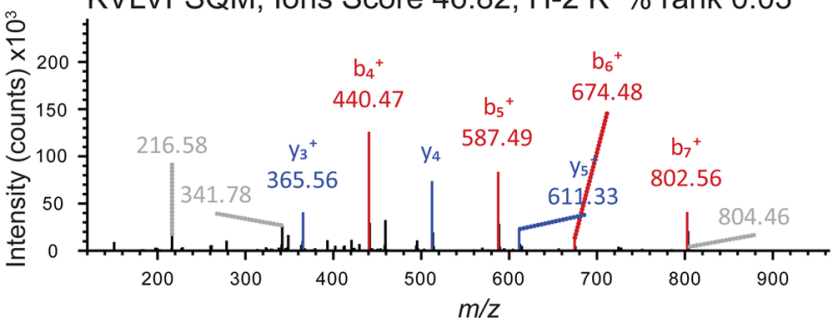

Figure 4. Examples of spectra assigned by only the MHC-DB search. Shown are several MS/MS spectra with high Mascot ions scores from the (A) B22.249 and (B) Y3 IPs that were not assigned to a peptide in the Parent-DB searches but assigned to peptides in the MHC-DB searches. Such peptides were mostly allotype-specific (B22.249 peptides were $\leq 2 \%$ NetMHC rank for the H-2 $\mathrm{D}^{\mathrm{b}}$ allele; Y3 peptides were $\leq 2 \%$ NetMHC rank for the $\mathrm{H}-2 \mathrm{~K}^{\mathrm{b}}$ allele).

HLA-Specific Database Searches Improve Statistical Power and Peptide Identifications in Human HLA-IP Searches

Having established the MHC-DB search approach in a mouse model, we next assessed the use of the targeted approach in human-derived samples. To test this approach, we retrieved eight MS/MS raw data files from recently published HLA ligandome data collected from peripheral blood mononuclear cells (PBMCs) of patients. ${ }^{27,28}$ We thus compiled targeted HLA databases for searching human HLA ligandome data based on the HLA-A and HLA-B allotypes of the patients. As in the mouse experiments, we began with a human reference proteome (Parent-DB) and used NetMHC to predict allotypespecific HLA peptides (NetMHC $\leq 2 \%$ rank) forming a targeted HLA database for each patient allotype (HLA-DB). Using a $2 \%$ rank cutoff, representation of predicted 8- to 11mer peptides was similar to that of the mouse databases (Supplementary Figure 7A). The Parent-DB search showed that most identified peptides were HLA binders (as revealed by a NetMHC rank $\leq 2 \%$ ) (Supplementary Figure 7B). Depending on the patient sample, Mascot searching against the HLA-DB resulted in 1.2- to 2.3-fold increases in HLA peptides compared with the Parent-DB (at 1\% FDR) (Figure 5A, Supplementary Data 2). As in the mouse experiments, peptide identification increases can also be attributed to better statistical power estimation because Percolator $q$ values were again lower for the HLA-DB search than for the Parent-DB search for all PBMC samples (Figure 5B). Using the HLA-DB searches also enabled the identification of peptides with lower Mascot ions scores on average (Figure 5C). The success of the targeted approach to increase HLA peptide identification in human samples is important because they may bear a host of important immunotherapeutic antigens.

MHC-I Peptides Assigned with the Targeted Databases Are Not Contaminant Peptides

Arguably, a potential pitfall of performing the MHC-targeted database search is that spectra identified as non-MHC-binding peptides (most likely contaminant peptides) could be falsely assigned as MHC-I peptides. The occurrence of these contaminant assignments can be discerned by performing a standard no enzyme search against the reference database. Examination of the PSM overlap between the Parent-DB and MHC-DB searches shows that few PSMs are solely identified by the Parent-DB (Supplementary Figure 8 ). In this regard, we found that only $12 / 118$ of B22.249 and 8/180 of those Y3 peptide spectrum matches (PSMs) considered nonbinders in the Parent-DB searches were reassigned as binders by the MHC-DB search (at 1\% FDR) (Figure 6A). In the human data, of the nonbinder PSMs from the Parent-DB search, again few were reassigned to HLA-binding peptides in the HLA-DB search (Figure 6B). These PSMs can be flagged for removal during routine practice of the targeted approach, and overall, the strategy still leads to a net increase in confident MHC-I or HLA peptide assignments. It is not known how much of this net increase comes at the cost of true MHC/HLA peptides not 

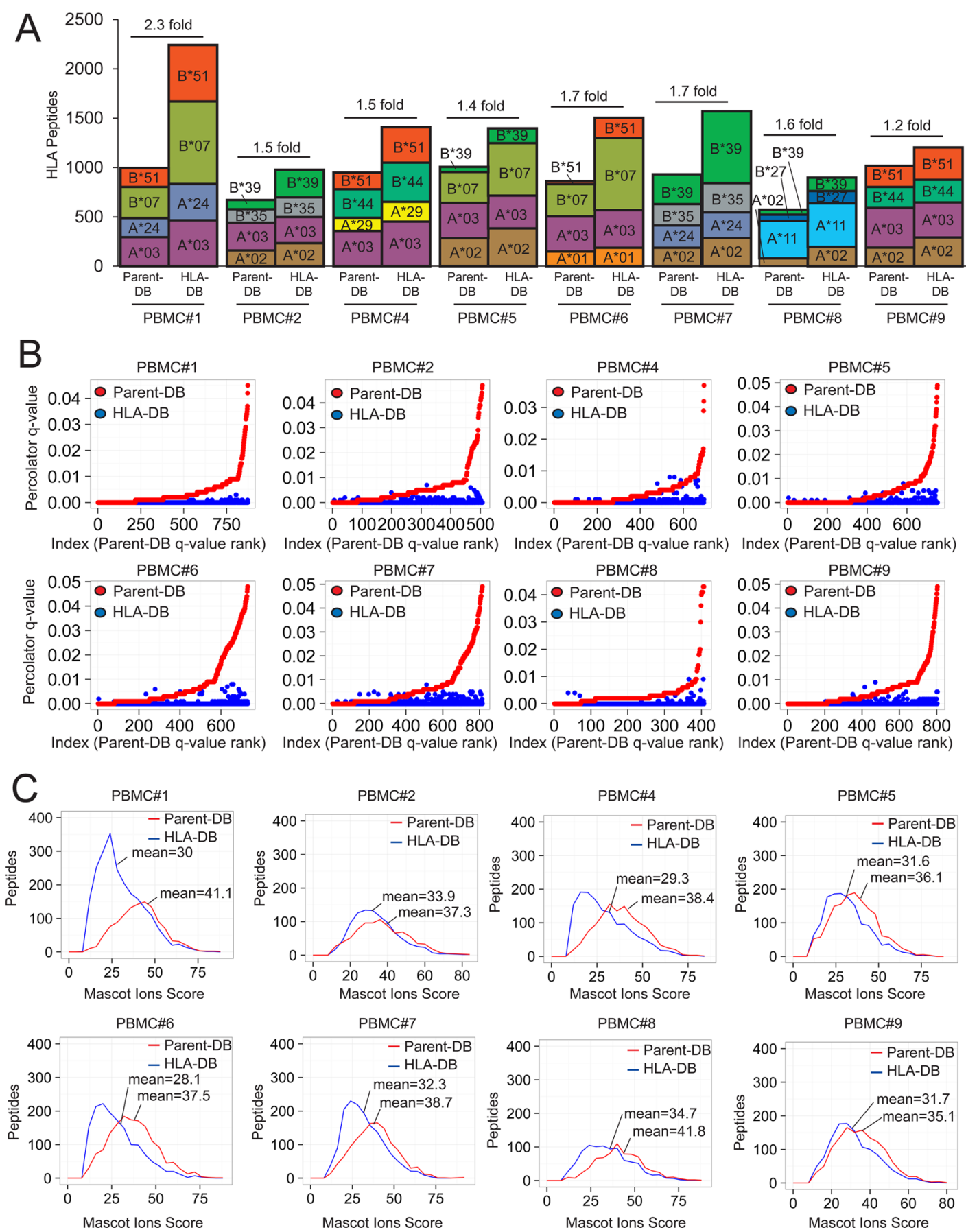

Figure 5. Targeted HLA database searches increase peptide identifications in human PBMC class I HLA ligandome data. Publically available human class I HLA ligandome raw data were retrieved and reanalyzed using searches based on allotype-specific targeted HLA databases. For each PBMC, HLA peptides were predicted from a human reference proteome database (Parent-DB) using NetMHC ( $\leq 2 \%$ rank) based on the patient allotype as stated in the publication. ${ }^{28} \mathrm{~A}$ patient-specific targeted HLA database search was performed and compared to the reference database (Parent-DB) using Mascot and Percolator. (A) Peptide identification increases (1\% FDR) for each PBMC data set comparing the targeted HLA database (HLADB) to the reference (Parent-DB) search. (B) For each PBMC ligandome search, Percolator $q$ values (up to 5\% FDR) are shown for peptides (all allotypes) identified using both the Parent-DB and HLA-DB search. Peptides are plotted in numerical order according to their $q$ value in the ParentDB search. Each PBMC number matches to that in the previously reported data set (Orbitrap data only). ${ }^{28}$ (C) Distribution and mean values of Mascot ions scores among identified peptides (1\% FDR) for the Parent-DB and HLA-DB search types for human PBMC ligandome data sets.

predicted by NetMHC, but true binders should be further interrogated by validation methods, iteratively improving the prediction tools. Such may be the case for HLA-C allotypes, which were not available from the PBMC data and are more poorly studied. Taken together with the maintenance of allotype specificity in the mouse experiment, these data also support the notion that non-MHC-I binders will not match the MHC-DB. We therefore propose that the targeted approach is a superior option for identifying MHC-I ligands from MS data as a result of its enhanced statistical power and will reveal otherwise unidentified ligands. The targeted approach could also bolster patient-specific immunotherapies because it is 
A B22.249-IP Parent-DB search non-binders (>2\% Rank) 53 peptides, 118 PSMs

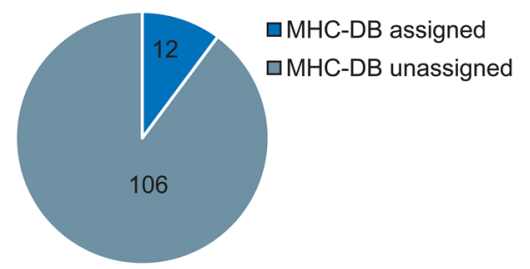

Y3-IP Parent-DB search non-binders (>2\% Rank) 93 peptides, 180 PSMs

B
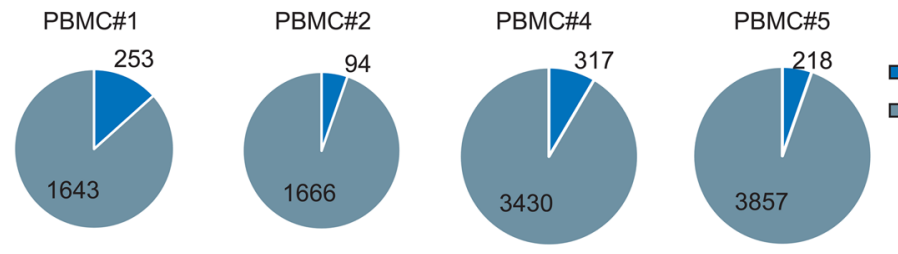

$\square \mathrm{MHC}-\mathrm{DB}$ assigned
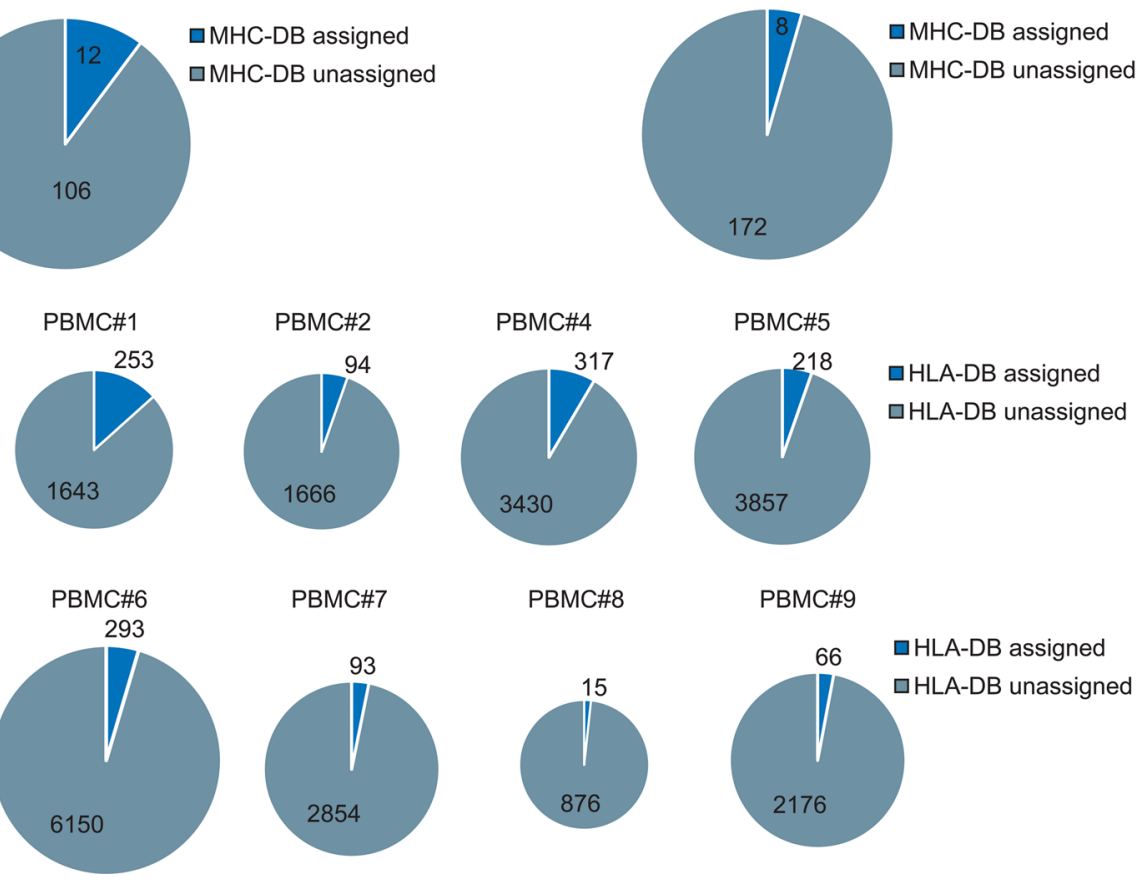

PBMC\#8

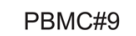

$\square$ HLA-DB assigned

$\square$ HLA-DB unassigned
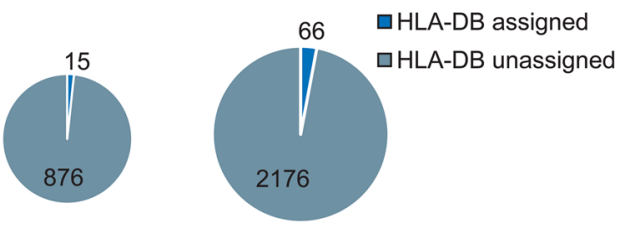

Figure 6. Most contaminant peptides are not assigned to MHC peptides. (A) Fraction of PSMs identified as nonbinders (NetMHC > 2\% rank) in the Parent-DB search that are reassigned as a binder by the MHC-DB search (1\% FDR). (B) Fraction of PSMs from each human PBMC ligandome identified as nonbinders (NetMHC > 2\% rank) in the Parent-DB search that are reassigned as a binder by the HLA-DB search (1\% FDR).

conceivable that targeted databases could be based on patient sequence data, improving the likelihood of detecting, for example, neoantigen peptides in cancer. ${ }^{29}$

\section{CONCLUSIONS}

MHC ligandomes hold information on therapeutic targets essential for understanding antigen-specific, T-cell-mediated immunity. Exciting recent developments in antitumor immunotherapy involving T-cell-based therapeutics, including PD-1 and CTLA4 immune checkpoint inhibitors, have led to a renewed importance for MHC-I ligand identification, ${ }^{30}$ with their potential for use as peptide vaccines. Database search strategies for identifying MHC ligand mass spectra from LCMS/MS experiments were initially intended to be employed by matching spectra to predictable protein fragments cleaved by proteolytic enzymes. ${ }^{31}$ This leads to a lack of enzymatic cleavage specificity in the search and is one of the many potential reasons why the success rate in assigning peptide identifications to ligand mass spectra data is low. Here, by compiling databases limited to predicted MHC-I peptides, we have greatly reduced the spectral search space. This approach essentially replaces the proteolytic peptides with predicted MHC-I ligands so that search strategies can be implemented as originally intended. As mentioned, unassigned spectra in MHCI ligand studies could result from IP of nonclassical ligands that our approach does not consider such as those proteasomally spliced, ${ }^{1}$ which have recently been introduced as being widespread. Furthermore, it is not known to what extent proteasomal splicing, peptide bulging, or post-translational modifications play in the ability of peptides to be predicted by NetMHC. It is worth noting that many newly discovered proteasomally spliced peptides were not considered binders by NetMHC ${ }^{1}$ but might be better predicted as more is known about them. Although novel peptide motifs missed as part of our predictive approach can be recovered by performing a complementary nontargeted search, caution should be taken in combining iterative searches unless a global FDR is properly considered. $^{32}$ Future approaches should address how to combine such searches. Overall, however, the targeted approach is effective for most scenarios, and as pan-allotype prediction tools are improving over time, ${ }^{33}$ targeted database searches will only improve. Furthermore, advances in MS speed and sensitivity, alongside better MHC-I IP methodologies, will result in the acquisition of even greater numbers of quality spectra. Finally, this novel targeted database search approach could be extended to other non-MHC-based investigations, such as using motif-targeted databases composed of known kinase phosphorylation motifs, thus improving phosphopeptide identification rates. We ultimately suggest that the targeted database search for MHC-I peptide identification is an essential step forward in the ever-expanding field of $\mathrm{MHC}$ ligand analysis.

\section{ASSOCIATED CONTENT}

S Supporting Information

The Supporting Information is available free of charge on the ACS Publications website at DOI: 10.1021/acs.jproteome.6b00971.

All identified mouse peptides. (XLSX)

All identified human peptides. (XLSX)

Supplementary Figure 1. Decoy database comparisons. Supplementary Figure 2. Search results at 5\% FDR. Supplementary Figure 3. Search results by NetMHC \% rank cutoff. Supplementary Figure 4. Predicted and Identified NetMHC \% ranks. Supplementary Figure 5. Percolator PEP statistics. Supplementary Figure 6 . 
Mascot ions score distributions. Supplementary Figure 7. Predicted and Identified HLA peptides. Supplementary Figure 8. Database overlap.(PDF)

\section{AUTHOR INFORMATION}

Corresponding Author

*Phone: 1-(902)-830-2952. Fax: 1-(902)-494-3292. E-mail: shashi.gujar@dal.ca.

ORCID (-)

Shashi Gujar: 0000-0002-5427-0829

Author Contributions

OJ.P.M. and P.K. contributed equally.

\section{Author Contributions}

J.P.M., P.K., H.S., D.J.K., D.C., Y.K., and S.G. conceived the approach and performed experiments. P.K. wrote the code for database compilation (SpectMHC). A.M.C. helped with instrumentation. J.P.M. and P.K. performed the data analysis. M.N. provided bioinformatics support. T.S., S.S., and P.W.L. provided input. J.P.M. and S.G. wrote the manuscript.

Notes

The authors declare no competing financial interest.

Mouse MS (.raw) files have been deposited to Chorus, https:// chorusproject.org, ID\#1098.

\section{ACKNOWLEDGMENTS}

We acknowledge Drs. Steve Gygi and Brian Erickson (Harvard Medical School) for providing feedback on the manuscript. We further acknowledge Dr. Dan Gaston (Nova Scotia Health Authority) for bioinformatics advice. This work was supported by grants from the Canadian Cancer Society Research Institute (CCSRI), Canadian Institutes of Health Research (CIHR), and Terry Fox Research Institute (TFRI) to S.G. and P.W.L.. D.C., Y.K., and T.S. are supported by the CIHR. J.P.M. is supported through the Cancer Research Training Program (CRTP) of BHCRI. D.C. was supported previously by CRTP from BHCRI and the Nova Scotia Health Research Foundation (NSHRF). P.K. is supported by Nova Scotia Graduate Scholarships fund.

\section{REFERENCES}

(1) Liepe, J.; Marino, F.; Sidney, J.; Jeko, A.; Bunting, D. E.; Sette, A.; Kloetzel, P. M.; Stumpf, M. P. H.; Heck, A. J. R.; Mishto, M. A large fraction of HLA class I ligands are proteasome-generated spliced peptides. Science 2016, 354 (6310), 354-358.

(2) Caron, E.; Kowalewski, D. J.; Koh, C. C.; Sturm, T.; Schuster, H.; Aebersold, R. Analysis of major histocompatibility complex (MHC) immunopeptidomes using mass spectrometry. Mol. Cell. Proteomics 2015, 14 (12), 3105-3117.

(3) Chen, D. S.; Mellman, I. Oncology meets immunology: the cancer-immunity cycle. Immunity 2013, 39 (1), 1-10.

(4) Yadav, M.; Jhunjhunwala, S.; Phung, Q. T.; Lupardus, P.; Tanguay, J.; Bumbaca, S.; Franci, C.; Cheung, T. K.; Fritsche, J.; Weinschenk, T.; et al. Predicting immunogenic tumour mutations by combining mass spectrometry and exome sequencing. Nature 2014, 515 (7528), 572-576.

(5) Kowalewski, D. J.; Schuster, H.; Backert, L.; Berlin, C.; Kahn, S.; Kanz, L.; Salih, H. R.; Rammensee, H.-G.; Stevanović, S.; Stickel, J. S. HLA ligandome analysis identifies the underlying specificities of spontaneous antileukemia immune responses in chronic lymphocytic leukemia (CLL). Proc. Natl. Acad. Sci. U. S. A. 2015, 112 (2), E166E175.

(6) Barnstable, C. J.; Bodmer, W. F.; Brown, G.; Galfre, G.; Milstein, C.; Williams, A. F.; Ziegler, A. Production of monoclonal antibodies to group A erythrocytes, HLA and other human cell surface antigens-new tools for genetic analysis. Cell 1978, 14 (1), 9-20.

(7) Laumont, C. M.; Daouda, T.; Laverdure, J.-P.; Bonneil, É.; Caron-Lizotte, O.; Hardy, M.-P.; Granados, D. P.; Durette, C.; Lemieux, S.; Thibault, P.; et al. Global proteogenomic analysis of human MHC class I-associated peptides derived from non-canonical reading frames. Nat. Commun. 2016, 7, 10238.

(8) Nagarajan, N. A.; de Verteuil, D. A.; Sriranganadane, D.; Yahyaoui, W.; Thibault, P.; Perreault, C.; Shastri, N. ERAAP shapes the peptidome associated with classical and nonclassical MHC class I molecules. J. Immunol. 2016, 197 (4), 1035-1043.

(9) Noble, W. S. Mass spectrometrists should search only for peptides they care about. Nat. Methods 2015, 12 (7), 605-608.

(10) Frewen, W. E.; Merrihew, G. E.; Wu, C. C.; Noble, W.S.; MacCoss, M. J. Analysis of peptide MS/MS spectra from large-scale proteomics experiments using spectrum libraries. Anal. Chem. 2006, $78,5678-5684$.

(11) Schubert, B.; Brachvogel, H.-P.; Jürges, C.; Kohlbacher, O. EpiToolKit-a web-based workbench for vaccine design. Bioinformatics 2015, 31 (13), 2211-2213.

(12) Lundegaard, C.; Lund, O.; Nielsen, M. Accurate approximation method for prediction of class I MHC affinities for peptides of length 8,10 and 11 using prediction tools trained on 9mers. Bioinformatics 2008, 24 (11), 1397-1398.

(13) Vita, R.; Overton, J. A.; Greenbaum, J. A.; Ponomarenko, J.; Clark, J. D.; Cantrell, J. R.; Wheeler, D. K.; Gabbard, J. L.; Hix, D.; Sette, A.; et al. The immune epitope database (IEDB) 3.0. Nucleic Acids Res. 2015, 43 (D1), D405-D412.

(14) Rammensee, H. G.; Bachmann, J.; Emmerich, N. P. N.; Bachor, O. A.; Stevanovic, S. SYFPEITHI: Database for MHC ligands and peptide motifs. Immunogenetics 1999, 50 (3-4), 213-219.

(15) Lin, H. H.; Ray, S.; Tongchusak, S.; Reinherz, E. L.; Brusic, V. Evaluation of MHC class I peptide binding prediction servers: Applications for vaccine research. BMC Immunol. 2008, 9, 8.

(16) Andreatta, M.; Nielsen, M. Gapped sequence alignment using artificial neural networks: Application to the MHC class I system. Bioinformatics 2016, 32, 511-517.

(17) Kowalewski, D. J.; Stevanović, S. Biochemical large-scale identification of MHC class I ligands. Methods Mol. Biol. 2013, 960, $145-157$.

(18) Rappsilber, J.; Ishihama, Y.; Mann, M. Stop and go extraction tips for matrix-assisted laser desorption/ionization, nanoelectrospray, and LC/MS sample pretreatment in roteomics. Anal. Chem. 2003, 75, 663-670.

(19) Haas, W.; Faherty, B. K.; Gerber, S. A.; Elias, J. E.; Beausoleil, S. A.; Bakalarski, C. E.; Li, X.; Villen, J.; Gygi, S. P. Optimization and use of peptide mass measurement accuracy in shotgun proteomics. Mol. Cell. Proteomics 2006, 5, 1326-1327.

(20) Shanmugam, A. K.; Nesvizhskii, A. I. Effective leveraging of targeted search spaces for improving peptide identification in tandem mass spectrometry based proteomics. J. Proteome Res. 2015, 14 (12), 5169-5178.

(21) Wang, X.; Slebos, R. J. C.; Wang, D.; Halvey, P. J.; Tabb, D. L.; Liebler, D. C.; Zhang, B. Protein identification using customized protein sequence databases derived from RNA-seq data. J. Proteome Res. 2012, 11, 1009-1017.

(22) Nielsen, M.; Andreatta, M. NetMHCpan-3.0; Improved prediction of binding to MHC Class I molecules integrating information from multiple receptor and peptide length datasets. Genome Med. 2016, 8, 33.

(23) Hämmerling, G. J.; Hämmerling, U.; Lemke, H. Isolation of twelve monoclonal antibodies against Ia and H-2 antigens. Serological characterization and reactivity with $\mathrm{B}$ and $\mathrm{T}$ lymphocytes. Immunogenetics 1979, 8 (1), 433-445.

(24) Hämmerling, G. J.; Rüsch, E.; Tada, N.; Kimura, S.; Hämmerling, $\mathrm{U}$. Localization of allodeterminants on $\mathrm{H}-2 \mathrm{~Kb}$ antigens determined with monoclonal antibodies and H-2 mutant mice. Proc. Natl. Acad. Sci. U. S. A. 1982, 79 (15), 4737-4741. 
(25) Käll, L.; Canterbury, J. D.; Weston, J.; Noble, W. S.; MacCoss, M. J. Semi-supervised learning for peptide identification from shotgun proteomics datasets. Nat. Methods 2007, 4 (11), 923-925.

(26) Elias, J. E.; Gygi, S. P. Target-decoy search strategy for increased confidence in large scale protein identifications by mass spectrometry. Nat. Methods 2007, 4, 207-214.

(27) Caron, E.; Espona, L.; Kowalewski, D. J.; Schuster, H.; Ternette, N.; Alpízar, A.; Schittenhelm, R. B.; Ramarathinam, S. H.; Lindestam Arlehamn, C. S.; Chiek Koh, C.; et al. An open-source computational and data resource to analyze digital maps of immunopeptidomes. eLife 2015, 4, e07661.

(28) Faridi, P.; Aebersold, R.; Caron, E. A first dataset toward a standardized community- driven global mapping of the human immunopeptidome. Data Brief 2016, 7, 201-205.

(29) Walz, S.; Stickel, J. S.; Kowalewski, D. J.; Schuster, H.; Weisel, K.; Backert, L.; Kahn, S.; Nelde, A.; Stroh, T.; Handel, M.; et al. The antigenic landscape of multiple myeloma: mass spectrometry (re)defines targets for T-cell-based immunotherapy. Blood 2015, 126 (10), $1203-1213$

(30) Coulie, P. G.; Van den Eynde, B. J.; van der Bruggen, P.; Boon, $\mathrm{T}$. Tumour antigens recognized by $\mathrm{T}$ lymphocytes: at the core of cancer immunotherapy. Nat. Rev. Cancer 2014, 14 (2), 135-146.

(31) Eng, J. K.; McCormack, A. L.; Yates, J. R. An approach to correlate tandem mass spectral data of peptides with amino acid sequences in a protein database. J. Am. Soc. Mass Spectrom. 1994, 5 (11), 976-989.

(32) Kertesz-Farkas, A.; Keich, U.; Noble, W. S. Tandem mass spectrum identification via cascaded search. J. Proteome Res. 2015, 14, 3027-3038.

(33) Karosiene, E.; Lundegaard, C.; Lund, O.; Nielsen, M. NetMHCcons: a consensus method for the major histocompatibility complex class I predictions. Immunogenetics 2012, 64 (3), 177-186. 